\section{External auditory feedback from covert oral behavior during silent reading*}

\author{
F. J. McGUIGAN† \\ Hollins College, Hollins College, Va. 24020
}

Ss were selected for high levels of covert oral behavior during silent reading. High speech-muscle amplitude during silent reading produced a slightly noxious tone, and a reduction in amplitude removed the tone. Feedback reduced amplitude of the controlling and associated speech muscles, but withdrawal of feedback returned the amplitude to about baseline level, indicating that the reduction was not permanent. More effective controlling techniques are required to determine stringently the function of the covert oral response with this approach.

There are two opposing views of the function of covert oral behavior during the performance of such linguistic tasks as silent reading: the traditional one that "subvocalization" is detrimental (e.g., Wood, 1966) and the alternative (recently revived) that the covert oral response facilitates the reading process (e.g., Edfeldt, 1960; McGuigan \& Rodier, 1968; McGuigan, 1970). One approach to help decide between these positions would be to decrease experimentally amplitude of covert oral behavior during silent reading and to observe the consequences on reading proficiency. First, however, we need to develop effective control over the covert oral response. External feedback from the speech musculature might serve for ihis purpose. To date, however, only sparse and inconclusive data have been reported on efforts to manipulate this response class (e.g., Hardyck, Petrinovich, \& Ellsworth, 1966; McGuigan, 1967 ; Hardyck \& Petrinovich, 1969).

\section{METHOD}

Initial screening produced six Ss with high levels of covert oral behavior during silent reading. Each $S$ was introduced to the laboratory, and sensors were attached to various oral and nonoral regions of the body (see below). Each day $S$ relaxed and then silently read a different passage standardized for his educational level. After a series of daily adaptation sessions of reading without feedback (three to seven, depending on the $S$ ),

* The project reported herein was performed pursuant to a grant from the U.S. Office of Education. Department of Health. Education, and - Welfare. The opinions express d herein, however. do not necessarily reflect the position or policy of the U.S. Office of Education, and no official endorsement by the U.S. Office of Education should be inferred.

-Special thanks to Betsy Adamson. Claudia Albright. Kathy Blake, Nancy Cummings, Douglas Gresham. Rebecca Hungerford, Dennis McRitchie, Madehne Shepperson. and particularly our Ss for helping with this research. auditory feedback signals from the speech musculature were introduced. During the feedback sessions (from 2 to 20, depending on the $S$ ), electromyographic (EMG) signals from the chin or lips automatically produced a slightly noxious tone (a potential punisher) when the S's response amplitude exceeded a specified level. A reduction of the response amplitude below that level terminated the tone (a potential negative reinforcer). The response amplitude that would produce feedback was specified for each $\mathrm{S}$ such that the tone would have been produced about $50 \%$ of the time words per minute (WPM) appear at the top. during the final no-feedback adaptation session. After the feedback sessions, the $S s$ read for a series of sessions (from 2 to 18 ) with feedback removed. The criterion for commencing feedback was that the amplitude of the response that was to produce feedback had become reasonably stabilized over sessions. EMGs were integrated and digitized for $10-\mathrm{sec}$ intervals during rest and during reading, and means for each were computed for each $S$ (cf. McGuigan, 1967, and McGuigan \& Rodier, 1968, for quantification details and for other information concerning the laboratory and procedures).

\section{RESULTS}

Each mean response amplitude during each prereading rest session was subtracted from the corresponding mean value during silent reading for that day. The psychophysiological results for S 1 (a female college student) are plotted as a function of session in the lower part of Fig. 1. No external feedback while reading was given during the first six sessions. Feedback from the chin was started during Session 7 , but the $\mathrm{S}$ was not told why the tone was initiated or terminated. The general increase in chin and tongue amplitude through Session 27 suggests that, without awareness of the function of the tone,



Fig. 1. EMG amplitudes for each session (lower). Feedback started when chin amplitude exceeded 20 microV during Sessions 7-33; thresholds for later sessions were lowered to 16 and 9 microV. Corresponding laboratory reading rates in 


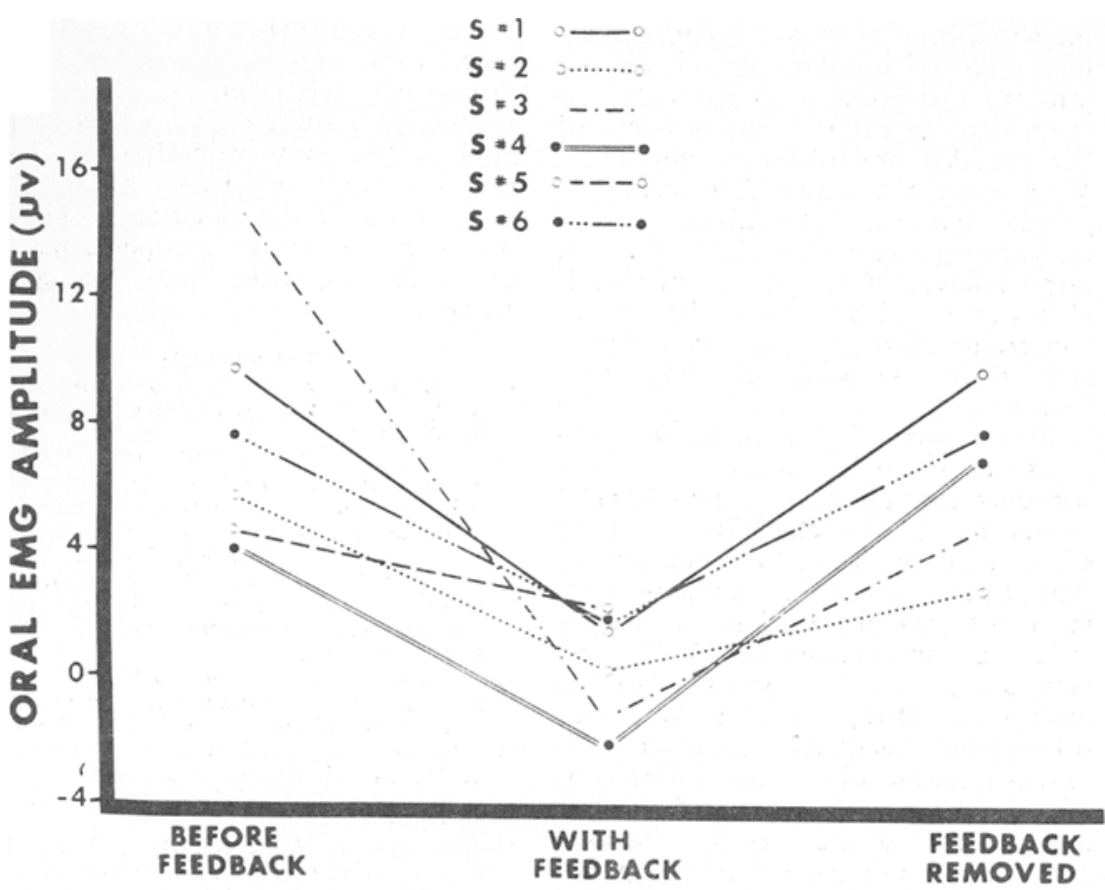

Fig. 2. Effect of feedback presentation and subsequent withdrawal on the triggering covert oral behavior during silent reading. Feedback was from the chin for Ss 1, 3, 4, and 5 and from the lips for Ss 2 and 6.

the $\mathrm{S}$ did not learn to reduce her covert oral behavior. At the start of Session 28 , the $S$ was told (to her amazement) that she controlled the tone, but she was not told how; a gradual decrease in amplitude of chin EMG then followed (Sessions 28-41). For Sessions 7-33, the tone came on when chin amplitude exceeded 20 microv (root-mean-square). For later sessions, the criterion was lowered to 16 and $9 \mathrm{microV}$, as specified in Fig. 1. Through this shaping procedure and by being aware that the tone served a feedback function, the $\mathbf{S}$ substantially lowered the level of her chin response. The accompanying decrease in tongue EMG suggests that the effect was generalized throughout the oral region.

Nonpreferred arm EMG was stable around zero throughout, indicating that S's originally heightened covert oral behavior during reading was not merely an aspect of general bodily activation; rather, the EMG increase was probably localized in the speech region. Lack of change in arm EMG as a function of feedback condition also suggests that feedback did not have a general disturbing effect and that consequent behavioral changes were localized in the oral region. Finally, when feedback was eliminated (Sessions 42 and 43), chin EMG dramatically increased to approximately the baseline level of Sessions 1-6, indicating that the continuous presence of feedback was subsequent nonfeedback reading sessions. clearly not inverse (usable tongue and rate measures were missing from Session 43).

The five additional Ss (two male and three female varying in ages between 7 and 19 years) were studied as was $S 1$, except that after the initial no-feedback adaptation sessions, they were told that the tone was indicative of excessive lip or chin movements and that their task was to prevent the tone's onset. Results (illustrative of the more extended curves) are summarized in Figs. 2 and 3 . The values plotted are for the last no-feedback session (when the $\mathbf{S}$ was well adapted to the laboratory), for the last feedback session (when feedback had become effective in removing the tone), and for the last session in which feedback was withdrawn. (The value for the last feedback session used for S 1 was under the "unaware" condition.) Figure 2 shows that feedback was always effective in decreasing the amplitude of the covert oral behavior (whether from chin or lips) during silent reading and that its withdrawal returned the behavior to approximately baseline level-the reduced amplitude of covert oral behavior during silent reading is not permanent, and when it did occur, it evidently was not merely due to instructions to reduce response amplitude. These results are thus at variance with the statements of Hardyck et al (1966) that reduction of speech muscle activity to resting levels

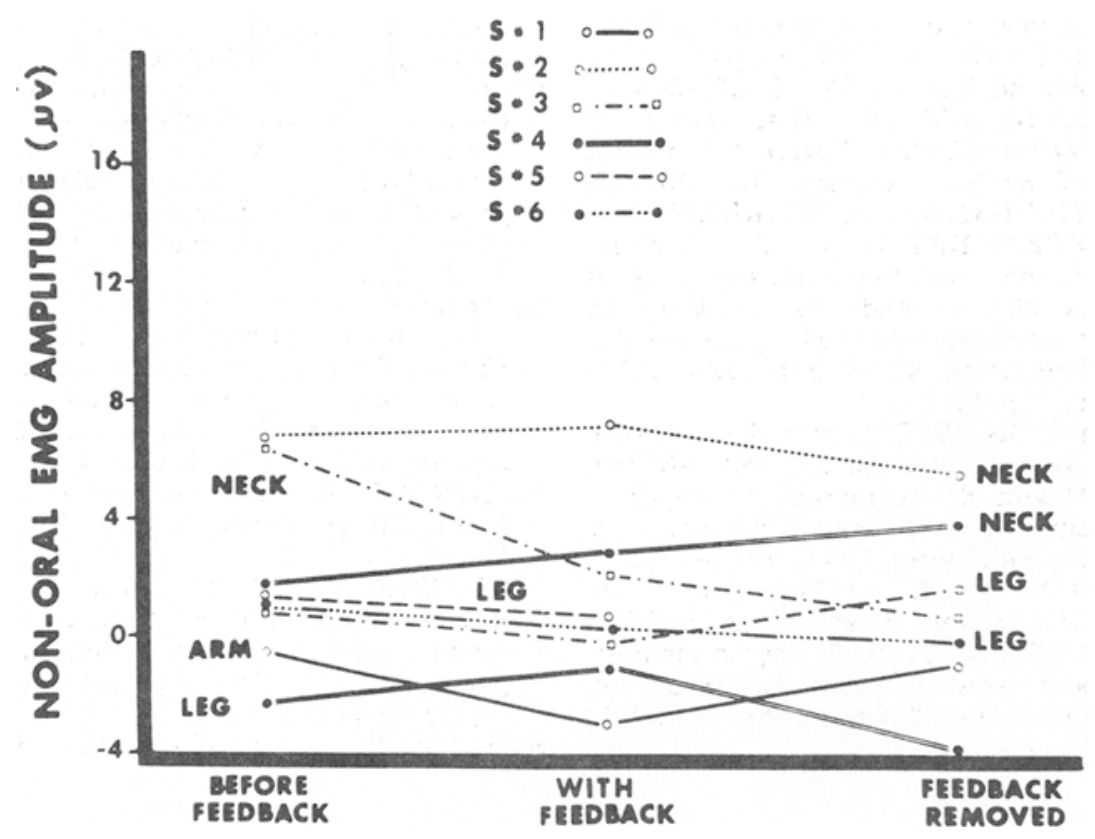

Fig. 3. Amplitude of nonoral response measures during feedback and 
is immediate (accomplished by most Ss within $5 \mathrm{~min}$ ) and long lasting.

The effect of feedback on other covert oral measures was essentially the same as for the one that triggered the feedback: feedback from the lips or chin also depressed associated muscle activity in the lips, chin, and tongue, and the accompanying oral measure typically increased once feedback was removed. The most extreme example was S 6, whose lips triggered feedback. The mean increase in chin amplitude from rest to silent reading for S6 was 24.7 microV before feedback; with feedback, the difference between resting and reading was $-3.0 \mathrm{microV}$, while when feedback was removed, the difference in mean chin amplitude increased to 8.1 microv.

Figure 3 shows that heightened covert oral behavior during silent reading is generally independent of nonoral measures and that the administration of feedback and its subsequent withdrawal have little systematic effect on these nonoral measures.

The introduction and subsequent withdrawal of feedback was about equally often accompanied by increased and decreased reading rates, measured as number of words per minute; this measure is probably too insensitive to reflect consequences of the reduced oral response amplitude. Mean comprehension scores during the prefeedback, feedback, and postfeedback sessions were, respectively, $69 \%, 66 \%$, and $72 \%$, showing that the Ss were comprehending about as well during and after feedback as they were before.

In conclusion, it appears that the presentation of a slightly noxious stimulus contingent on a high level of covert speech muscle activity during silent reading, and the cessation of that stimulus contingent on a decrease in covert oral behavior, is effective in reducing amplitude of both the controlling and associated speech muscles. But, as indicated by subsequent feedback removal, the reduced amplitude is not permanent. The heightened level of covert behavior during silent reading appeared to be localized in the speech region, and nonoral regions sampled were not systematically affected by feedback condition. Covert oral behavior can thus be only temporarily controlled through feedback techniques as used here. A more prolonged reduction in amplitude would be required before we could apply a "response-reduction" strategy to stringently test alternative hypotheses of the function of covert oral behavior during the performance of such linguistic tasks as silent reading.

\section{REFERENCES}

EDFELDT, A. W. Silent speech and silent reading. Chicago: University of Chicago Press, 1960.

HARDYCK. C. D.. \& PETRINOVICH, L. F. Treatment of subvocal speech during reading. Journal of Reading, 1969, 12 , $361-368,419-422$.

HARDYCK, C. D.. PETRINOVICH, L. F.. $\&$ ELLSWORTH, D. W. Feedback of speech muscle activity during sirent reading: Rapid extinction. Science, 1966. 154. $1467-1468$.

MCGUIGAN. F. J. Feedback of speech muscle actirity during silent reading: Two comments. Science, $1967,157,579-580$.

McGUIGAN. F. J. Covert oral behavior during the silent performance of language tasks. Psychological Bulletin, 1970. 74. 309-326.

MeGUIGAN. F. J., \& RODIER, W. I.. III. Effects of auditory stimulation on covert oral behavior during silent reading. Joumal of Experimental Psychology, $1968,76,649-655$.

WOOD, E. W. Reading dynamics. U.S.A: Evelyn Wood Reading Dynamics. 1966

\section{CURRENT LITERATURE ON HUMAN HEARING}

AARONSON, D. (New York University, New York, N.Y. 1003), MARKOWITZ, N., \& SHAPIRO, H. Percpetion and immediate recall of normal and "compressed" auditory sequences. Perception \& Psychophysics, 1971, 9, 338-344.

BUCHSBAUM, M. (Laboratory of Mental Health, National Institutes of Health, Bethesda, Md. 20014), SILVERMAN, J., HENKIN, R. I., \& PFEFFERBAUM, A. Contrast effects on the auditory evoked response and its relation to psychophysical judgments. Perception \& Psychophysics, 1971, 9, 379-384.

DAWSON, W. E. (University of Notre Dame, Notre Dame, Ind. 46556). Magnitude estimation of apparent sums and differences. Perception \& Psychophysics, 1971, 9 368-374.

DOOST, R. (University of Connecticut, Storrs, Conn. 06268), \& TURVEY, M. T. Iconic memory and central processing capacity. Perception \& Psychophysics, 197I, 9, 269-274.
FOSS, D. J. (University of Texas at Austin, Austin, Tex. 78712), \& DOWELL, B. E. High-speed memory retrieval with auditorily presented stimuli. Perception \& Psychophysics, 1971, 9, 465-468.

GREEN, D. M. (University of California at San Diego, La Jolla, Calif. 92037), \& LUCE, R. D. Detection of auditory signals presented at random times: III. Perception \& Psychophysics, 1971, 9, 257-268.

LaBERGE, D. (University of Minnesota, Minneapolis, Minn. 55455). On the processing of simple visual and auditory stimuli at distinct levels. Perception \& Psychophysics, 1971, 9, 331-334.

RUBINSTEIN, L. (Psychiatric Epidemiology Research Unit, New York, N.Y. 10032 ), \& GRUENBERG, E. M. Intramodal and crossmodal sensory transfer of visual and auditory temporal patterns. Perception \& Psychophysics, 1971, 9, 385-390.

SCHULMAN, A. I. (University of
Virginia, Charlottesville, Va. 22901). Detectability of the deletion of a tone from a tone-plus-noise background. Perception \& Psychophysics, 1971, 9, 496-498.

TREISMAN, A. M. (Department of Experimental Psychology, South Parks Road, Oxford, England), \& FEARNLEY, S. Can simultaneous speech stimuli be classified in parallel? Perception \& Psychophysics, 1971, 9, 1-7.

WARREN, R. M. (University of Wisconsin-Milwaukee, Milwauke, Wis. 53201). Identification times for phonemic components of graded complexity and for spelling of s peech. Perception \& Psychophysies, 1971, 9, 345-349.

WEERTS, T. (University of Wisconsin, Madison, Wis. 53706$)$ \& THURLOW, W. R. The effects of eye position and expectation on sound localization. Perception \& Psychophysics, 1971, 9, 35-39. 